\title{
Random Walker Segmentation based Tag Completion for Image Retrieval
}

\author{
Shrikant Badghaiya \\ Department of Computer Science \\ OIST \\ Bhopal, India
}

\begin{abstract}
Image retrieval is a technique of accessing texts or images from the web. Although there are various techniques implemented for the image retrieval such as using content based or tag based. Hence by using the technique for the image retrieval can be used in various fields. Tag based Image retrieval is a technique also used for the efficient retrieval of images [1]. Although the technique is efficient but it provides less accuracy, hence for the better access of the image retrieval based on tags segmentation is done and then matrix is generated to classify the images and hence can be retrieved in more accurate manner.
\end{abstract}

\section{Key Terms}

Tag Completion, Segmentation, Random Walk, Automatic Annotations, CBIR, TBIR.

\section{INTRODUCTION}

Image processing is a technique of accessing and process images so that it can be used for a variety of applications. Image retrieval provides accessing of images in a better way so that images can be classified easily. Content bases image retrieval is a technique used for the access of certain features such as histogram based or color correlogram based. But content based image retrieval doesn't provide efficient access of images hence accuracy is less.

Tag based image retrieval is a new technique of retrieving of images which provides high accuracy as compared to the content based image retrieval. Tag matrix completion uses the concept of providing a tag to the image and hence a matrix is generated which contains 1 if the image contains tag otherwise 0 .

Although the tag matrix completion is efficient technique as compared to the content based image retrieval techniques, but it only provides accuracy about $90 \%$. Hence a new and efficient technique based on segmentation is implemented using random walker segmentation.

Here the tag matrix is implemented after applying random walker segmentation which increases the image retrieval accuracy of $95 \%$.

\section{Random Walker Segmentation}

The technique is used for the image segmentation which uses the concept of background and foregrounds seed pixels. The process is running iteratively until the segmentation is done. Initially red and blue pixels are set on the image object part and non-object part. The pixels can be traversed with its neighbors until background pixels find.

\author{
Atul Barve \\ Department of Computer Science \\ OIST \\ Bhopal, India
}

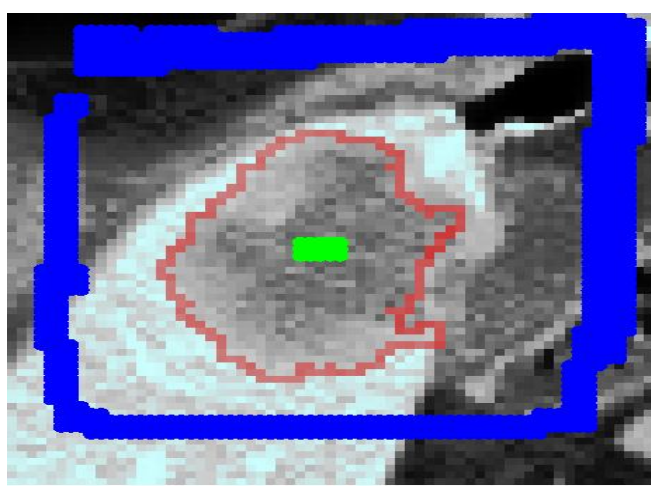

Figure 1. Example of Random Walker Segmentation

Tag based Image Retrieval

Tagging is a way to identify certain area or piece of data in various images along with proper retrieval. Tagging is an important concept which can be used for the image sharing and for the management among users. By providing tags to describe the content of photos, many manipulations can be easily accomplished, such as indexing, browsing, and search [2]. Intuitively, the most convenient approach to generate tags is to investigate automatic tagging (or annotation) techniques [3], [4] However, although great advances have been achieved in automatic tagging, currently these methods can hardly obtain satisfactory performance for real-world photos that contain significantly varying content. Automatic image tagging (also known as image annotation) methods that aim to classify the images with respect to a set of semantic concepts can be used as an intermediate stage. Since Image retrieval using Tagging can be used for the high level access of the images.

\section{LITERATURE SURVEY}

Since there are various techniques implemented for the retrieval of images such as Content based retrieval [6]. Since light weight methods are used for the better retrieval of images as compared to the retrieval using SVM [5].

1. Relevance feedback can be viewed as a way of supplying multiple example images by involving users in the retrieval process [7]. Some computing techniques used in relevance feedback, where multiple selected images are handled, could be adapted to process images supplied as examples in multi-example queries, for example, the weight optimization method [8]. In this approach, some difference should be considered in developing retrieval schemes with multiexample queries. In conventional CBIR schemes with relevance feedback [7], the user provides a single image as a query and the system returns the initial retrieval results. A session labeling returned images requires several iterations 
In contrast, the retrieval schemes with multi-example queries aim to search relevant images using multiple example images provided by the user as a query in a session with no iterations. From the sampling point of view when only relevant example images are concerned, the approaches of relevance feedback and multi-example querying are different. The approach of obtaining relevant example images using relevance feedback can be viewed as a kind of cluster sampling, because the labeled relevant images would be clustered around the initial query image in some way which is determined by the search strategy of CBIR systems. However, the approach of multi-example querying can be viewed as a kind of simple random sampling, as the example images provided by the user have certain diversity in low-level feature spaces [9].

Supervised image classification is beneficial for CBIR while the predefined classes and the training samples are available. A multi-class classifier would be trained to classify images in the collection into the predefined classes. Given a query, the system would predict a relevant image class and return the top ranked images in the relevant class as the retrieval results. Therefore, image classification could speed-up image retrieval in large collections and improves retrieval accuracy [10].

The existing image classification approaches could be based on low-level modeling or high-level modeling for image content [11. In contrast, in high-level modeling, image content is modeled by high-level features such as visual words [12]. A method is proposed for image classification based on approximate global geometric correspondence using local descriptors [13].

Image can be classified in two ways or stages i.e generative based approach and discriminative based approach. The discriminative approach, such as SVM, can directly optimize classification boundaries and organize images into classes according to their decision values. Zhang et al. propose a method to combine SVM and KNN for effective image classification, which can overcome the drawbacks of any single classification algorithm [15]. In contrast to the discriminative approach, the generative approach estimates the density of images in each image class and uses Bayes formula to compute the posterior. This approach is able to incorporate background knowledge easily and is naturally suit to multiple classes. Image retrieval can also be done using the combinatorial method of classification using Bayesian and density estimation [14].

\section{PROPOSED METHODOLOGY}

The proposed methodology implemented here consists of following phases:

1. Take an input training corel dataset.

2. Apply Random Walker Segmentation on each of the training dataset.

3. Assign Tag to each of the training dataset images.

4. Automatically assign tags to each of the testing corel dataset based on the stored features of the trained images.

5. Retrieve the images using TBIR.

\section{RESULT ANALYSIS}

The table shown below is the dataset used for the analysis of the image retrieval. The corel dataset contains 1500 images of various categories in which each of the image contains 5 different tags.
Table 1. Corel Dataset Description

\begin{tabular}{|c|c|}
\hline Parameters & Corel Dataset \\
\hline No. of Images & 1500 \\
\hline No. of Tags per Image & 5 \\
\hline No. of Images per Tag & $100-150$ \\
\hline
\end{tabular}

The table shown below is the Analysis of the proposed work. Here for the analysis 5 tags are used such as church, tiger, animal, water and design. The proposed methodology provides about $95-98 \%$ accuracy of the image retrieval.

Table 2. Analysis of the proposed work

\begin{tabular}{|c|c|c|c|}
\hline & \multicolumn{3}{|c|}{ Proposed Work } \\
\hline Tag & Precision & Recall & F-Score \\
\hline 1 & 96.17 & 95.37 & 95.76832933 \\
\hline 2 & 97.12 & 95.67 & 96.38954718 \\
\hline 3 & 98.75 & 97.28 & 98.00948834 \\
\hline 4 & 99.81 & 96.34 & 98.04430691 \\
\hline 5 & 99.37 & 96.32 & 97.82123154 \\
\hline
\end{tabular}

The table shown below is the Analysis of the Existing work. Here for the analysis 5 tags are used such as church, tiger, animal, water and design. The Existing technique provides about $85-93 \%$ accuracy of the image retrieval which is comparatively less.

Table 3. Analysis of the Existing Work

\begin{tabular}{|c|c|c|c|}
\hline & \multicolumn{3}{|c|}{ Existing Work } \\
\hline Tag & Precision & Recall & F-Score \\
\hline 1 & 93.53 & 90.44 & 91.95904985 \\
\hline 2 & 95.32 & 91.25 & 93.24060674 \\
\hline 3 & 98.16 & 92.74 & 95.37305815 \\
\hline 4 & 99.74 & 94.32 & 96.95431104 \\
\hline 5 & 97.16 & 93.28 & 95.18047469 \\
\hline
\end{tabular}

The figure shown below is the comparison of precision of the existing and proposed methodology. The proposed methodology provides more precision than existing work.

\section{Comparison of Precision}

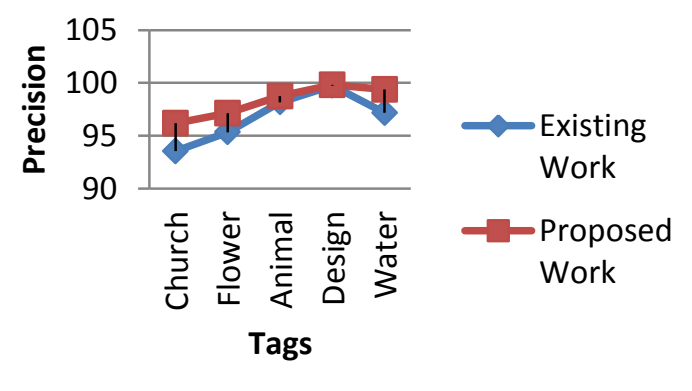

Figure 2. Comparison of Precision 
The figure shown below is the comparison of Recall of the existing and proposed methodology. The proposed methodology provides more Recall than existing work.

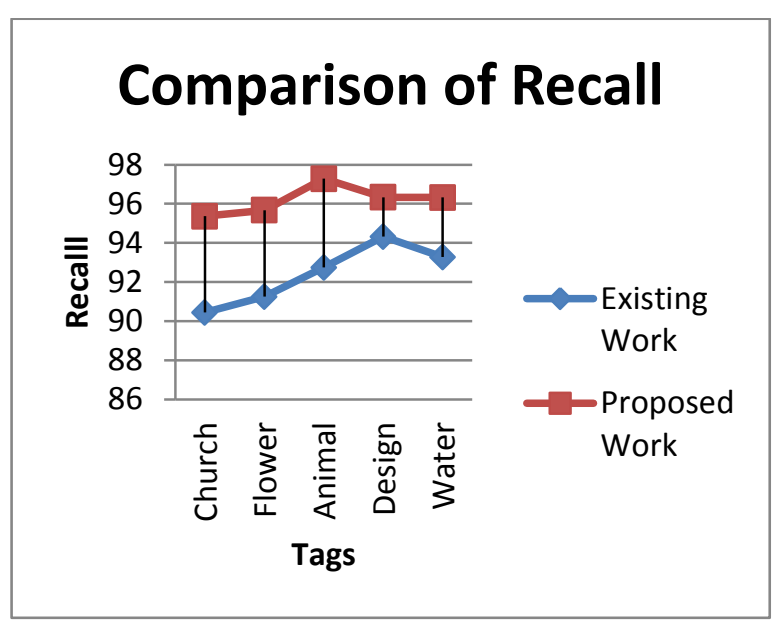

Figure 3. Comparison of Recall

The figure shown below is the image retrieval from corel dataset for the tag "Tiger".

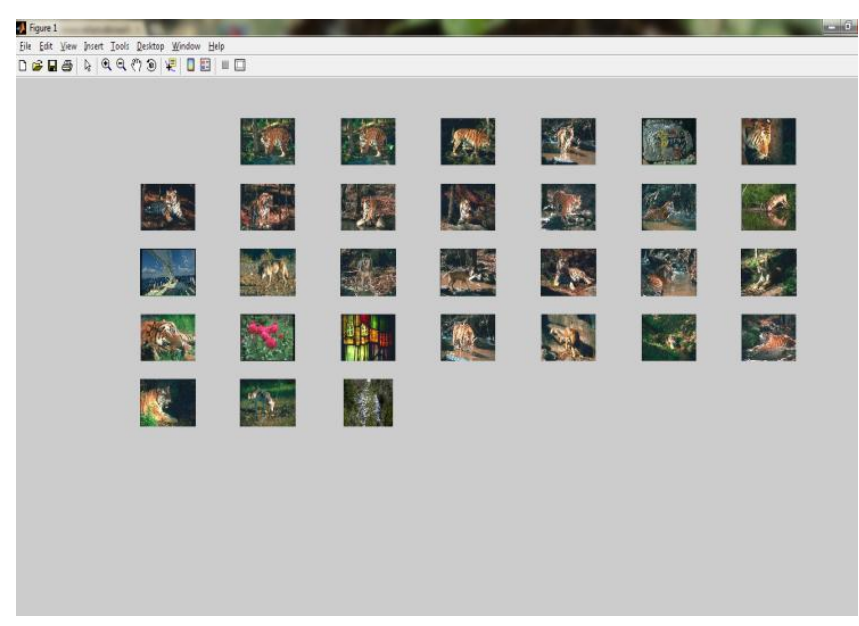

Figure 4. Image Retrievla using "Tiger" Tag

The figure shown below is the image retrieval from corel dataset for the tag "Flower".

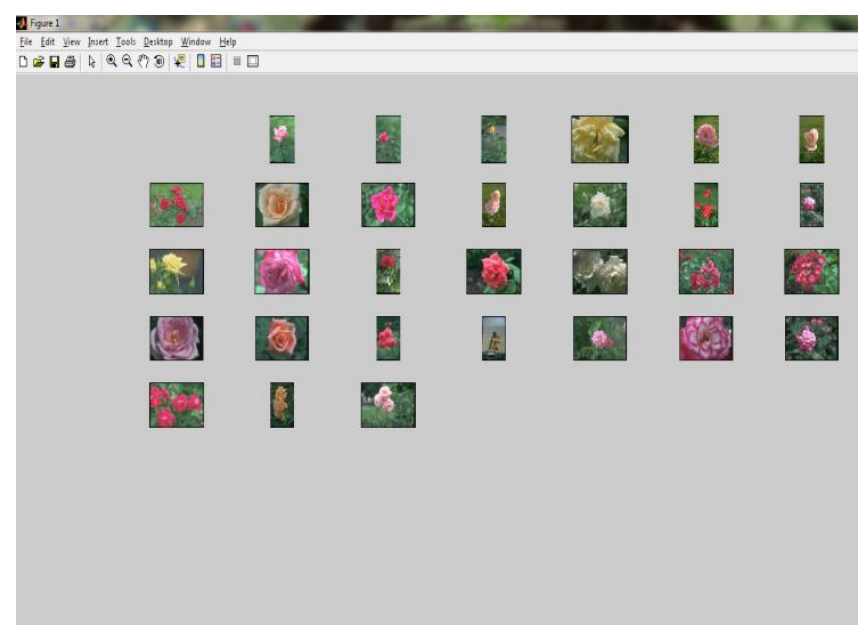

Figure 5. Image Retrieval using "Flower" Tag
The figure shown below is the image retrieval from corel dataset for the tag "Church".

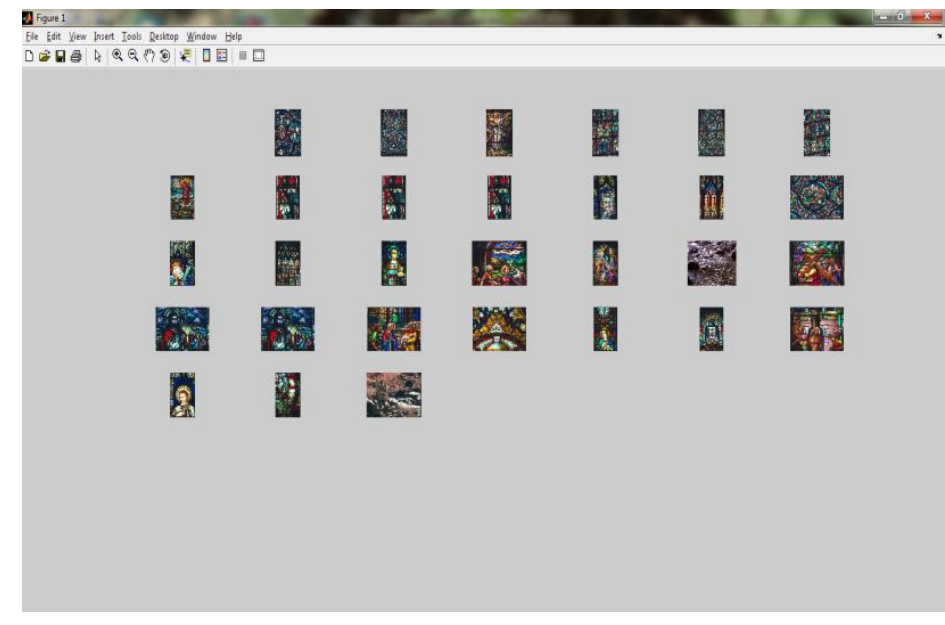

Figure 6. Image Retrieval using “Church" Tag

The figure shown below is the image retrieval from corel dataset for the tag "Water".

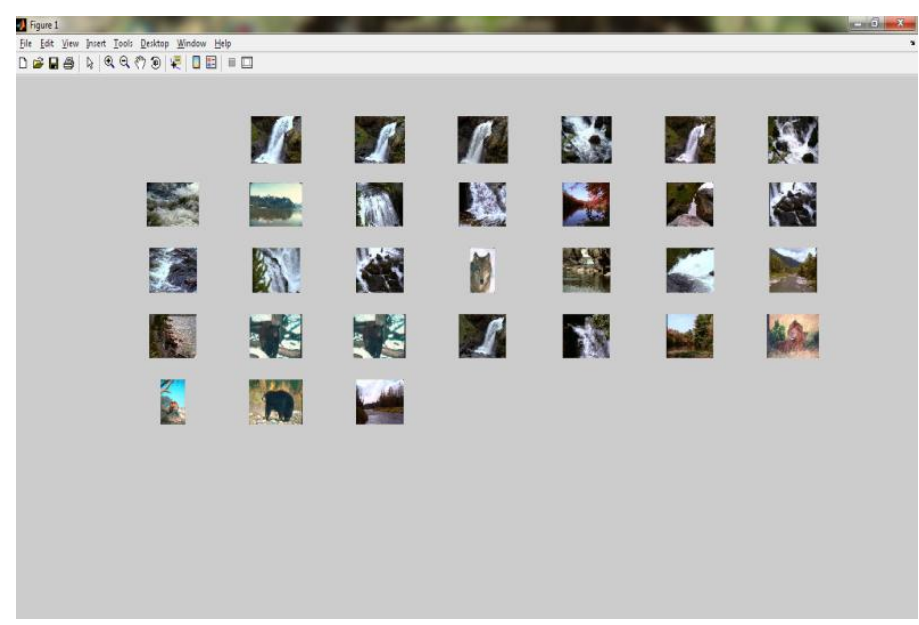

Figure 7. Image Retrieval using "Water" Tag

The figure shown below is the image retrieval from corel dataset for the tag "Design".

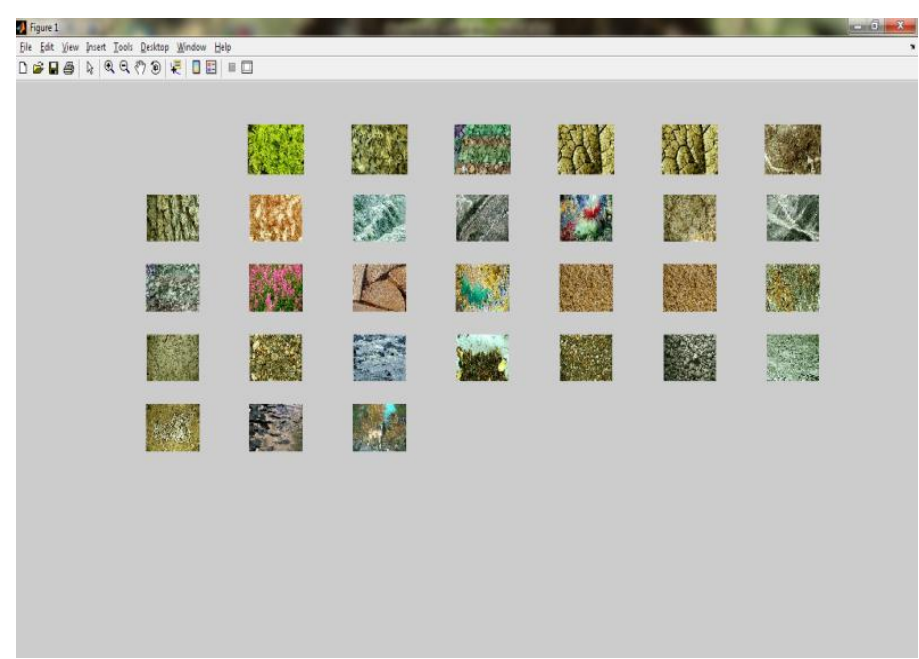

Figure 8. Image Retrieval using “Design" Tag 


\section{CONCLUSION}

The result analysis shows the performance of the proposed methodology. The previous technique implemented for the tag based image retrieval provides accuracy only about $85-90 \%$. However the proposed technique implemented using image segmentation based image retrieval provides accuracy of about $98 \%$. The analysis is done on the corel dataset which contains 1500 images of nearly 15 categories; each category contains about 100 images of 5 tags per image. The proposed technique provides accuracy on almost all tags for images.

\section{FUTURE WORK}

Although the technique is efficient for the image retrieval but further enhancements can be done for all types of images using some supervised learning approach and hence can be applied for all types of image databases.

\section{REFERENCES}

[1] Lei Wu, Rong Jin, and Anil K. Jain,” Tag Completion for Image Retrieval", IEEE Transaction on Pattern Analysis and Machine Intelligence, 2013.

[2] Lei Wu, Rong Jin and Anil K. Jain "Tag Completion for Image Retrieval”, IEEE Transactions On Pattern Analysis And Machine Intelligence, Vol. 35, No. 3, pp. 716 - 727, March 2013.

[3] J. Li and J. Wang, "Real-time computerized annotation of pictures", IEEE Transactions On Pattern Analysis And Machine Intelligence, vol. 30, no. 6, pp. 985-1002, Jun. 2008.

[4] J. Jeon, V. Lavrenko, and R. Manmatha, "Automatic image annotation and retrieval using cross-media relevance models," in Proc. 21 ${ }^{\text {st }}$ Annu. Int. ACM SIGIR Conf. Research and Development in Information Retrieval, pp. 119-126, 2003.

[5] N. Abbadeni. An approach based on multiple representations and multiple queries for invariant image retrieval. In International Conference on Advances in Visual Information Systems, pages 570-579, Shanghai, China, June 2007.
[6] A. P. Natsev and J. R. Smith. "Active selection for multiexample querying by content" In IEEE International Conference on Multimedia and Expo, pages 445 - 448, Baltimore, USA, July 2003.

[7] X. S. Zhou and T. S. Huang. Relevance feedback in image retrieval: A comprehensive review. Multimedia Systems, 8(6):536-544, April 2003.

[8] Y. Rui and T. S. Huang. Optimizing learning in image retrieval. In IEEE International Conference on Computer Vision and Pattern Recognition, pages 1236-1243, Hilton Head, USA, June 2000.

[9] X. Y. Jin and J. C. French. Improving image retrieva effectiveness via multiple queries. Multimedia Tools and Applications, 26(2):221-245, June 2005.

[10] R. Datta, D. Joshi, J. Li, and J. Z. Wang. Image retrieval: Ideas, influences, and trends of the new age. ACM Computing Surveys, 40(2):5:1-5:60, 2008.

[11] A. Bosch, X. Munoz, and R. Marti. Which is the best way to organize/classify images by content? Image and vision computing, 25(6):778-791, June 2007.

[12] A. Bosch, A. Zisserman, and X. Munoz. Scene classification via plsa. In European Conference on Computer Vision, pages 517-530, Graz, Austria, May 2006.

[13] S. Lazebnik, C. Schmid, and J. Ponce. Beyond bags of features: Spatial pyramid matching for recognizing natural scene categories. In IEEE International Conference on Computer Vision and Pattern Recognition, pages 21692178, New York, USA, June 2006.

[14] A. Vailaya, M. A. T. Figueiredo, A. K. Jain, and H. J. Zhang. Image classification for content-based indexing. IEEE Transactions on Image Processing, 10(1):117- 130, January 2001

[15] H. Zhang, A. C. Berg, M. Maire, and J. Malik. SVM-KNN: Discriminative nearest neighbor classification for visual category recognition. In IEEE International Conference on Computer Vision and Pattern Recognition, pages 21262136, New York, USA, June 2006. 